\title{
A rare benign disorder mimicking metastasis on radiographic examination: a case report of osteopoikilosis
}

\author{
Ali Erhan Ozdemirel · Burcu Duyur Cakit • \\ Hatice Rana Erdem • Bunyamin Koc
}

Received: 28 May 2010/ Accepted: 14 November 2010/Published online: 1 December 2010

(C) The Author(s) 2010. This article is published with open access at Springerlink.com

\begin{abstract}
Osteopoikilosis (OPK) is a rare, autosomal dominant bone disorder, characterized by multiple, discrete round or ovoid radio densities scattered throughout the axial and appendicular skeleton. OPK is usually asymptomatic but rarely there may be slight articular pain and joint effusions. OPK is generally diagnosed incidentally on radiographic examinations and may mimic different bone pathologies, including bone metastases. Radionuclide bone scan has a critical role in distinguishing OPK from osteoblastic bone metastases. In this case report, we present a young man with right hip pain due to OPK, whose plain radiogram and computerized tomography findings thought cancer metastasis.
\end{abstract}

Keywords Osteopoikilosis - Computerized tomography · Sclerotic bone lesions · Metastasis

\section{Introduction}

Osteopoikilosis (OPK) is an asymptomatic, rare bone dysplasia characterized by an abnormality in the enchondral bone maturation process [1]. Prevalence has been

A. E. Ozdemirel - B. D. Cakit - H. R. Erdem

2nd Department of Physical Medicine and Rehabilitation,

Ankara Training and Research Hospital, Ministry of Health, 06340 Cebeci, Ankara, Turkey

B. Koc

Department of Physical Medicine and Rehabilitation, University of Cumhuriyet, 58140 Sivas, Turkey

A. E. Ozdemirel $(\bowtie)$

1429. Cadde, 1462. Sokak 12/9,

06520 Cukurambar, Ankara, Turkey

e-mail: erhanozdemirel@hotmail.com estimated to be $1 / 50,000$. Studies of familial occurrence indicate an autosomal dominant pattern of genetic transmission. Sporadic forms are also presented. OPK can be noted at any age, and incidence is similar in both sexes [2]. Bone lesions are symmetric, numerous, small, welldefined, homogeneous and circular or ovoid. These small round and ovoid radiopacities in the juxtaarticular regions of bone are characteristic radiologic signs of OPK [3, 4]. The most frequently involved sites are the epiphyses and metaphyses of long tubular bones and the carpal bones, tarsal bones, pelvis and scapulae [1]. Laboratory findings and bone scintigraphy are usually normal.

OPK has occasionally been reported in association with skin manifestations, urogenital defects, rheumatic and skeletal disorders, organ anomalies, endocrine dysfunction, and dental and facial abnormalities [3, 5]. The main challenge lies in differential diagnosis from other condensing bone diseases, particularly osteoblastic metastases.

This rare hereditary condition is usually noticed radiologically as an incidental finding of diagnostic sclerotic lesions. OPK is usually asymptomatic, but in $15-20 \%$ of patients, there may be slight articular pain and joint effusions. In this case report, we present a young man with right hip and low back pain due to OPK, whose radiographic examination findings thought cancer metastasis initially.

\section{Case report}

A 35-year-old man was admitted to our outpatient clinic with pain on the right hip for 2 months. His pain was constant and deteriorated while walking. He did not have any systemic disease, and he was not taking medication regularly. There was no history of trauma, morning 


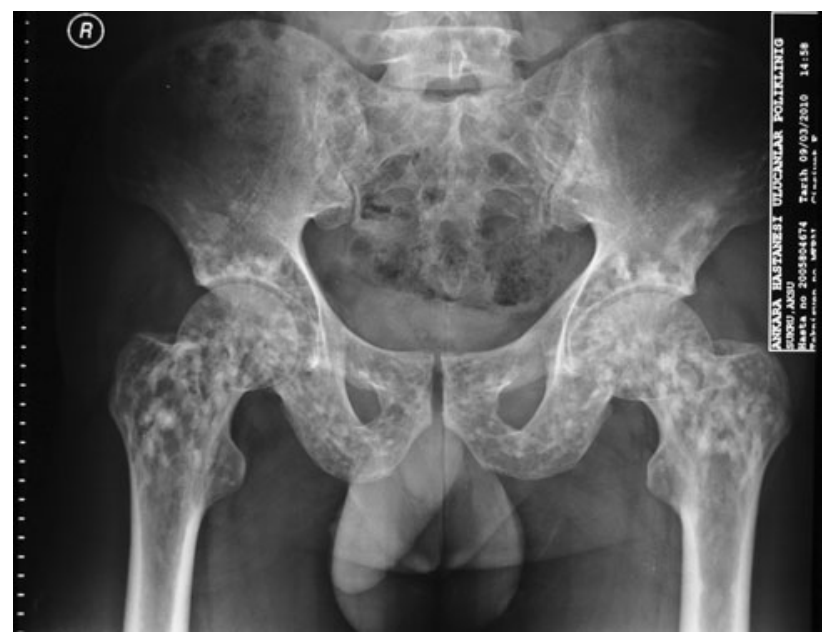

Fig. 1 Anteroposterior radiograph of the pelvis showing multiple, small, ovoid sclerotic lesions in both femoral heads, ileum and around the acetabulum of the pelvis, without narrowing the joint spaces

stiffness, weight loss and fever. On musculoskeletal examination, range of motion (ROM) of all joints was within normal limits, and neurological examination was also normal. ROM of the right hip was painful at the end of the range. No skin lesions were detected. Complete blood count, erythrocyte sedimentation rate, rheumatoid factor, C-reactive protein, thyroid, kidney and liver function tests, serum calcium, phosphorus, magnesium, alkaline phosphatase, 25[OH] vitamin D and parathyroid hormone levels were normal.

Pelvis radiogram revealed numerous small round foci of bony sclerosis on the upper heads of the femur and acetabulum (Fig. 1). Computerized tomography (CT) examination of pelvis confirmed multiple round sclerotic nodular lesions located in both iliac wings and sacrum, which were mimicking metastases (Fig. 2). Whole body bone scintigraphy was performed to rule out metastases. Tc-99m bone scintigraphy revealed normal findings. But incidentally kidney fusion anomaly was observed in scintigraphic images (Fig. 3). This anomaly was confirmed with urinary system ultrasonography as well. OPK was suspected from these findings, wrist radiogram which revealed multiple symmetric foci of dense radio-opaque spots in the metaphyses of distal radius and ulna, carpal bones, metacarpals and phalanges confirmed the diagnosis (Fig. 4). These radio-opaque spots are also detected on shoulder radiogram (Fig. 5).

In the light of these clinical and radiologic findings, the patient was diagnosed as OPK. Nonsteroidal anti-inflammatory drug was prescribed for his pain. After 1 month, he had no more pain at his hip and he became completely asymptomatic.

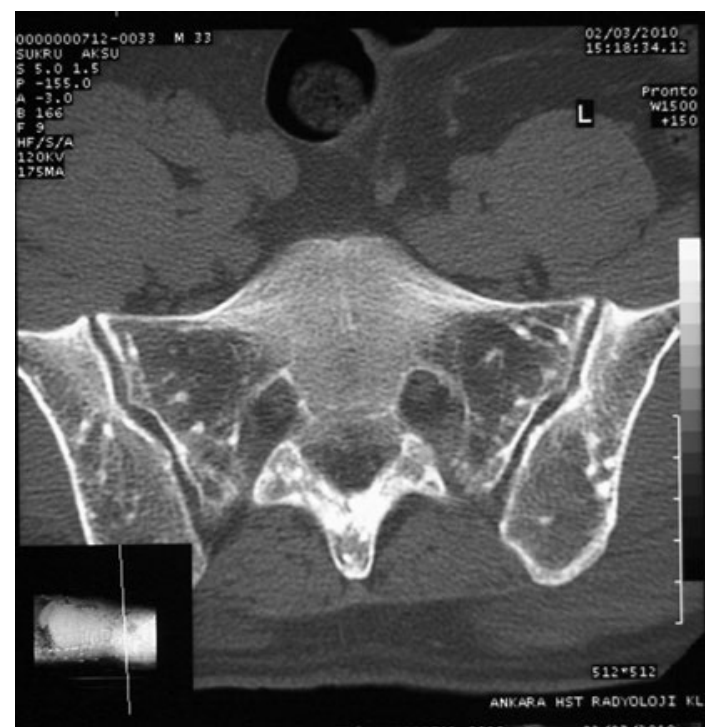

Fig. 2 Computed tomography image of the patient showing multiple round sclerotic nodular lesions at iliac wings and sacrum which are mimicking metastases

\section{Discussion}

OPK is a rare, inherited sclerosing bone dysplasia caused by failure of resorption of secondary spongious bone. The disorder consists of multiple benign bone islands scattered throughout the axial and appendicular skeleton. The bone islands typically clustered around the larger joints $[2,6]$.

OPK results in numerous $2-10 \mathrm{~mm}$ densities of oval or rounded shape that are distributed within the metaphyses and epiphyses of the long bones which appears in childhood and persists throughout life. The lesions are noted in a fairly symmetric distribution, especially around the knee and shoulder, along with the pelvis, carpal and tarsal bones. The lesions are less common in the skull, ribs, vertebral bodies and mandible [4, 7]. On histology, these foci are formed by dense trabeculae of spongious bone, sometimes forming a nidus without communication with the bone marrow [4, 6]. In our patient, carpal bones, metacarpals, distal radius and ulna, femur, pelvis and shoulder were involved.

The cause and the pathogenesis of OPK are not known, but there are many hypotheses: (1) A hereditary failure to form normal trabeculae along lines of stress. (2) Dysplasia of endochondral ossification, affecting secondary spongiosa, is characterized by errors in resorption or remodeling of secondary spongiosa, which results in focal densities and/or striations within the trabecular bone. (3) OPK is a bone manifestation of a generalized fibroproliferative or stenosing disease. It was confirmed that loss-of-function mutations in the LEMD3 gene could result in OPK. (4) An altered osteogenesis may be responsible for the lesions. 
Fig. 3 Tc-99m bone scintigraphy image of the patient showing kidney fusion anomaly



Fig. 4 Anteroposterior radiograph of the hands demonstrating multiple sclerotic foci in the distal radius and ulna, carpal, metacarpal and proximal phalangeal bones

It has been suggested that chronic abnormal remodeling of bone predisposed to malignant transformation. Probably due to active osteogenesis in OPK, coexistence of osteosarcoma and OPK is reported $[8,9]$.

Differential diagnoses include osteoblastic metastasis, tuberous sclerosis, mastocytosis and synovial chondromatosis [1,3]. Its major clinical significance is that it should

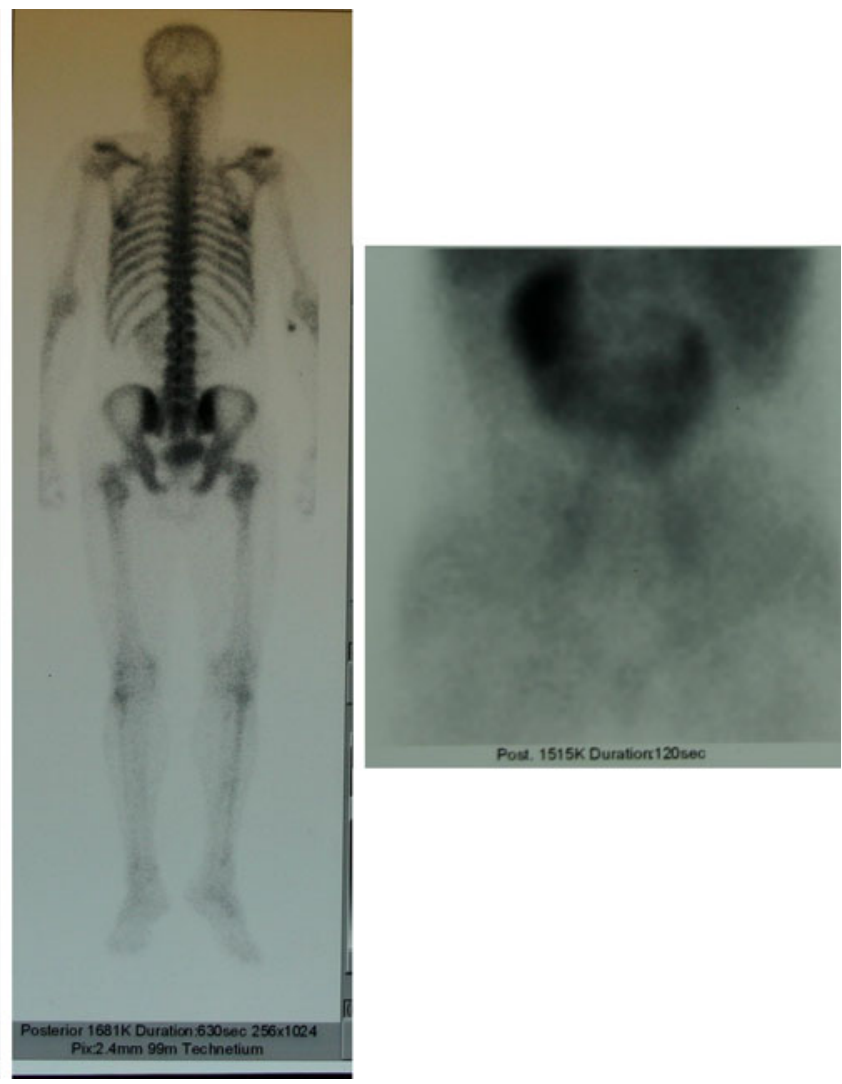

not be confused with metastatic osteoblastic lesions. Osteoblastic metastases are asymmetric and varied in size. Also a predilection for axial skeleton, osseous destruction, periostal reaction and positive scintigraphic findings, differentiates osteoblastic metastases from OPK [10]. In patients with a known or suspected primary malignancy, radionuclide bone scan has a critical role in distinguishing OPK from osteoblastic bone metastases. Typically, there is no increased uptake of radiopharmaceutical substance on nuclear medicine bone scan in OPK. This is contrary to the bone scan appearance of the most important differential diagnosis, osteoblastic bone metastases, which characteristically produce numerous "hot spots" of increased activity $[10,11]$. In both mastocytosis and tuberous sclerosis, symmetry, metaphyseal and epiphyseal preference, and uniform, well-defined foci are less striking than in OPK [1]. Although the findings in pelvis CT of our patient had been reported as osteoblastic metastases, bone scintigraphy for whole body with Tc-99m excluded the existence of metastatic lesions. It was suggested that, the definitive diagnosis of OPK made by radiography of both hands, since in all OPK patients, the metacarpal bones are affected [3]. Characteristic lesions detected on hand radiographs were also confirmed the diagnosis of OPK in our patient.

OPK can occur either as an isolated anomaly or in association with other abnormalities, mostly with skin lesions. In $\% 25$ of cases, patients have connective tissue 
Fig. 5 Bilateral anteroposterior radiograph of the shoulder showing multiple radiodense sclerotic foci of the proximal humerus

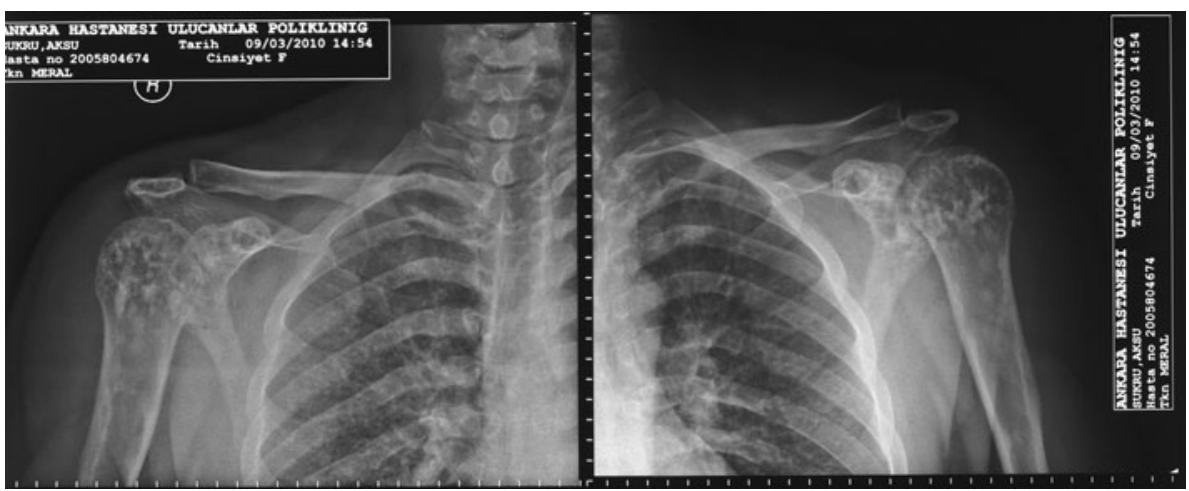

nevi called dermatofibrosis lenticularis disseminata (DLD). Overlapping of OPK and DLD was defined as BuschkeOllendorff syndrome $[1,12]$. OPK has also been found to be associated with a tendency to keloid formation, dwarfism, spinal stenosis, dystocia and melorheostosis as a result of proliferation of metabolically active fibroblasts due to the mutations of LEMD3 gene [7, 9]. Several rheumatologic pathologies have been reported to coexist with OPK, such as rheumatoid arthritis, lupus erythematosus, ankylosing spondylitis and familial Mediterranean fever [13]. None of these rheumatologic diseases were present in our case. Various developmental malformations have been reported also to be associated with OPK, including: coarctation of the aorta, double ureter, pubertas praecox, urogenital defects, growth abnormalities, exostoses, osteitis condensans ilii, Klippel-Feil syndrome, dacryocystitis, giant cell tumor, fibrous dysplasia, chondrosarcoma, osteosarcoma, synovial chondromatosis, facial abnormalities, dental abnormalities, and plantar and palmar keratomas [14]. We observed kidney fusion anomaly in our patient.

In conclusion, OPK is usually an asymptomatic condition and is usually found radiologically as an incidental finding, but sometimes there may be mild articular pain as in our patient. As in our case, when multiple, symmetric, round sclerotic lesions found on radiographic examinations, OPK should be kept in mind to avoid misdiagnosis, especially from osteoblastic metastases. We thought that due to the association of various malformations and risk of malign transformation with OPK, follow-up of OPK patients is essential.

Open Access This article is distributed under the terms of the Creative Commons Attribution Noncommercial License which permits any noncommercial use, distribution, and reproduction in any medium, provided the original author(s) and source are credited.

\section{References}

1. Resnick D, Niwayama G (1998) Enostosis, hyperostosis and periostitis. In: Reznick D, Niwayama G (eds) Diagnosis of bone and joint disorders. W.B Saunders Company, Philadelphia, pp 4084-4088

2. Serdaroglu M, Capkin E, Ucuncu F, Tosun M (2007) Case report of a patient with osteopoikilosis. Rheumatol Int 27:683-686

3. Benli IT, Akalin S, Boysan E, Mumcu EF, Kiş M, Türkoğlu D (1992) Epidemiological, clinical and radiological aspects of osteopoikilosis. J Boint Surg 74:504-506

4. Khot R, Sikarwar JS, Gupta RP, Sharma GL (2005) Osteopoikilosis: a case report. Ind J Rad Imag 15:453-454

5. Bicer A, Tursen U, Ozer C, Kaya TI, Dusmez D, Ikizoglu C (2002) Coexistence of osteopoikilosis and discoid lupus erythematosus: a case report. Clin Rheumatol 21:405-407

6. Resnick D, Niwayama G (1995) Enostosis, hyperostosis and periostitis. In: Resnick D (ed) Diagnosis of bone and joint disorders, 3rd edn. WB Saunders, Philadelphia, pp 4404-4411

7. Sarralde A, Garcia CD, Nazara Z (1994) Osteopoikilosis: report of a familial case. Genet Couns 5(4):373-375

8. Vanhoenacker FM, De Beuckeleer LH, Van Hul W et al (2000) Sclerosing bone dysplasias: genetic and radioclinical features. Eur Radiol 10:1423-1433

9. Couto AR, Bruges-Armas J, Peach CA et al (2007) A novel LEMD3 mutation common to patients with osteopoikilosis with and without melorheostosis. Calcif Tissue Int 81:81-84

10. Seron MA, Yochum TR (1996) Essentials of skeletal radiology. Williams \& Wilkins, Baltimore, pp 630-633

11. Appenzeller S, Castro GR, Coimbra IB (2007) Osteopoikilosis with abnormal bone scan: long-term follow-up. J Clin Rheumatol 13:291-292

12. Al Attia HM, Sherif AM (1998) Buschke-Ollendorf syndrome in a grande multipara: a case report and short review of the literature. Clin Rheumatol 17(2):172-175

13. Ureten K (2007) Osteopoikilosis in a patient with rheumatoid arthritis complicated with dry eyes. Rheumatol Int 27:1079-1082

14. Kaparov A, Uludag M, Sari H, Akarirmak U (2009) De Quervain's syndrome associated with osteopoikilosis: a case report and review of the literature. Rheumatol Int [Epub ahead of print]. doi:10.1007/s00296-009-1239-2 